\title{
Intervention on the left main coronary artery. Importance of periprocedural and follow-up intravascular ultrasonography guidance
}

\author{
Paweł Tyczyński, Zbigniew Chmielak, Jerzy Pręgowski, Marek Rewicki, Maciej Karcz \\ Department of Interventional Cardiology and Angiology, Institute of Cardiology, Warsaw, Poland
}

Postep Kardiol Inter 2014; 10, 2 (36): 130-132 DOI: $10.5114 /$ pwki.2014.43522

\begin{abstract}
A bstract
Periprocedural intravascular ultrasonography guidance for left main coronary artery stenting is well established. However, the role of this tool is also important at follow-up interventions. We present a case of a patient with previous history of left main coronary artery angioplasty. During a recent attempt to treat tight stenosis in the left anterior descending coronary artery, it was not possible to advance the stent into the left main coronary artery. Intravascular ultrasonography explained the difficulties encountered.
\end{abstract}

Key words: intravascular ultrasonography, percutaneous coronary intervention.

\section{Case report}

A 62-year-old male patient with stable coronary artery disease (CCS II), hypertension and paroxysmal atrial fibrillation was admitted to our department for further treatment.

His previous history included several percutaneous coronary interventions $(\mathrm{PCl})$. Six years ago $\mathrm{PCl}$ of the right coronary artery and the left circumflex artery (LCX) was performed.

Four years ago the patient underwent interventional treatment for unstable angina. The $\mathrm{PCl}$ of both the left main coronary artery (LMCA) and the left anterior descending coronary artery (LAD) were performed; a $4.5 \mathrm{~mm}$ $\times 24 \mathrm{~mm}$ bare metal stent (BMS, Driver, Medtronic, Minneapolis, USA) was implanted into the LMCA and a $3.5 \mathrm{~mm} \times$ $16 \mathrm{~mm}$ BMS (Liberte, Boston Scientific, USA) was deployed into the proximal LAD segment.

Two weeks before current admission to our department, the patient was hospitalized for unstable angina. Control angiography done in another hospital revealed tight stenosis in the proximal segment of the LAD and $80 \%$ stenosis in the distal LCX (Figure $1 \mathrm{~A}$ ). The operator decided to implant a stent in the LAD and to treat the LCX at the second stage. Using an EBU guide catheter and subsequently an AL guide catheter (Launcher, Medtronic, USA) in order to improve the catheter position, only balloon angioplasty of the LAD was possible to perform.
Several attempts to insert the stent into the LMCA were unsuccessful (Figure 1 C). As an acceptable angiographic result with non-significant residual stenosis in the LAD was achieved (Figure $1 \mathrm{D}$ ), the procedure was stopped.

Because of effort angina, the patient was referred to our department for further treatment. Repeated angiography showed a good result in the LAD. Nevertheless, a significant lesion in the LCX remained. In order to elucidate the previous problems with stent insertion, intravascular ultrasonography (IVUS) was attempted. However, it was not possible to insert the IVUS probe into the stented LMCA. The IVUS probe could not negotiate through the LMCA ostium and remained outside the stent lumen, in the sinus of Valsalva (Figure $1 \mathrm{E}$ ). Therefore IVUS could only visualize the part of stent protruding from the LMCA into the aorta. Most likely the wire entered the protruding LMCA stent through the stent cells from its abluminal site. Attempts to insert the wire into the lumen of the stented LMCA failed. In order to accurately assess the length of the protruding stent part, computed tomography angiography (CTA) was done. It showed high LMCA take-off from the sinus of Valsalva. The upper stent's surface was touching the sino-tubular junction and the lower stent's surface protruded up to $9 \mathrm{~mm}$ into the Valsalva sinus (Figure $1 \mathrm{~F}$ ). However, control angiography done 9 months later for unstable angina, revealed tight re-stenosis in proximal segment of LAD.

\section{Corresponding author:}

Paweł Tyczyński MD, PhD, Department of Interventional Cardiology and Angiology, Institute of Cardiology, 42 Alpejska St, 04-628 Warsaw, Poland, phone: +48 696091079 , e-mail: medykpol@wp.pl

Received: 14.11 .2013 , accepted: 20.01.2014 


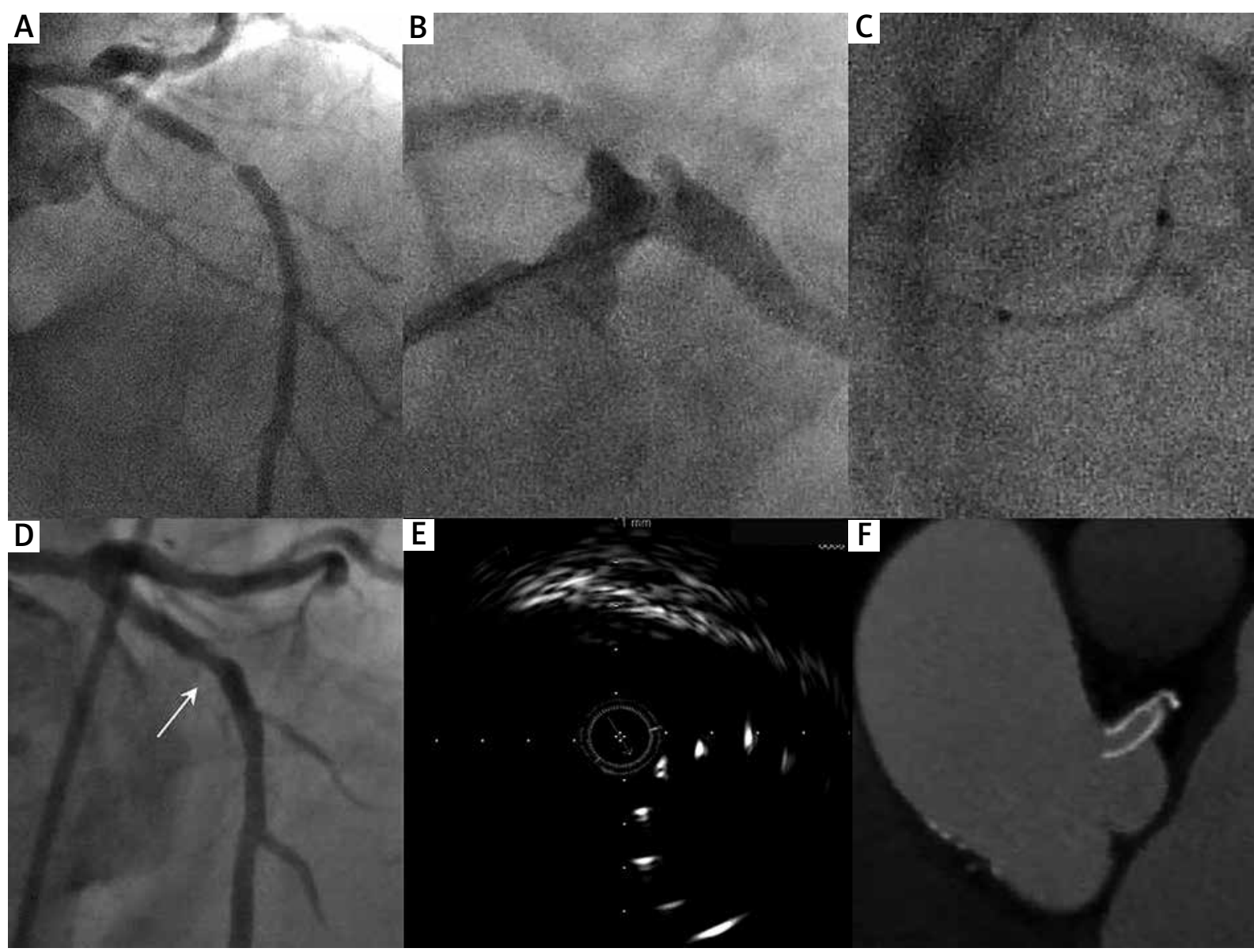

Figure 1. A - Tight stenosis in proximal LAD, B - non-axial LMCA cannulation, $\mathbf{C}$ - stent flexion during forced attempt of its insertion into the LMCA, D - residual non-significant stenosis (arrow) 2 weeks after balloon angioplasty, $\mathbf{E}$ - IVUS probe outside the lumen of the protruding LMCA stent, F - CTA image showing $9 \mathrm{~mm}$ protrusion of the lower surface of the proximal LMCA stent into the Valsalva sinus

CTA - computed tomography angiography, IVUS - intravascular ultrasonography, LAD - left anterior descending coronary artery, LMCA - left main coronary artery

Subsequently, the patient underwent successful surgical revascularization with the left internal mammary artery grafted to LAD and the saphenous vein graft sewn to LCX.

\section{Discussion}

Periprocedural IVUS guidance for optimal stent deployment and improvement of the LMCA stenting outcome is well established [1]. In our case IVUS examination was not done during initial LMCA stenting 4 years ago. Additionally, IVUS was not utilized during a recent, unsuccessful attempt of stent insertion. We can hypothesize that during recent balloon angioplasty the guidewire could not penetrate into the lumen of the partially protruding stent into the Valsalva sinus, but entered through the stent cell from its abluminal side. The false guidewire course could also be suspected from the suboptimal LMCA cannulation (Figure $1 \mathrm{~B}$ ). Although Driver stent is characterized by small, closed cells, it was possi- ble to pass a $4.0 \mathrm{~mm}$ balloon and perform balloon angioplasty in the LAD.

Thus, the role of IVUS should not be limited to the periprocedural optimization of LMCA stenting. Exact assessment of the possible stent protrusion should also be then performed. This information may be critical for possible interventions in the future, as in our case.

The CTA plays a complementary role to invasive coronary imaging, and in some cases might be of decisive value [2]. This refers to both follow-up assessment and pre-interventional strategy planning.

\section{Conclusions}

In rare cases angiography may not be enough for good assessment of ostial lesions. This especially refers to follow-up assessment after interventional treatment. The IVUS and CTA play complementary roles in elucidating the procedural difficulties. 


\section{References}

1. Park SJ, Kim YH, Park DW, et al. MAIN-COMPARE Investigators. Impact of intravascular ultrasound guidance on long-term mortality in stenting for unprotected left main coronary artery stenosis. Circ Cardiovasc Interv 2009; 2: 167-77.

2. Van Mieghem CA, Cademartiri F, Mollet NR, et al. Multislice spiral computed tomography for the evaluation of stent patency after left main coronary artery stenting: a comparison with conventional coronary angiography and intravascular ultrasound. Circulation 2006; 114: 645-53. 